\section{$\underset{\substack{\text { hommes } \\ \text { \& migrations }}}{ }$}

\section{Hommes \& migrations}

Revue française de référence sur les dynamiques

migratoires

$1311 \mid 2015$

Femmes et migrations

\title{
Bande dessinée et immigrations : un siècle d'histoire(s)
}

\section{Mikaël Petitjean}

\section{(2) OpenEdition \\ Journals}

\section{Édition électronique}

URL : http://journals.openedition.org/hommesmigrations/3337

DOI : 10.4000/hommesmigrations.3337

ISSN : 2262-3353

\section{Éditeur}

Musée national de l'histoire de l'immigration

\section{Édition imprimée}

Date de publication : 1 juillet 2015

Pagination : 144-146

ISBN : 978-2-919040-32-2

ISSN : $1142-852 X$

\section{Référence électronique}

Mikaël Petitjean, « Bande dessinée et immigrations : un siècle d'histoire(s) », Hommes \& migrations [En ligne], 1311 | 2015, mis en ligne le 09 février 2016, consulté le 15 septembre 2020. URL : http:// journals.openedition.org/hommesmigrations/3337 


\section{BANDE DESSINÉE ET IMMIGRATIONS UN SIĖCLE D'HISTOIRE(S)}

par MIKAËL PETITJEAN, chargé de mission au Musée national de l'histoire de l'immigration.

I Musée national de l'histoire de l'immigration

Lprésente jusqu'en septembre 2020 une exposition itinérante, Bande dessinée et immigrations: un siècle d'histoire(s), consacrée aux croisements entre l'histoire de l'immigration et l'histoire de la bande dessinée.

Le Musée national de l'histoire de l'immigration poursuit une réflexion amorcée lors de l'exposition Albums. Un siècle d'histoire dans la bande dessinée (2013). Au-delà de la diversité des approches esthétiques, l'histoire de la bande dessinée est marquée depuis ses débuts par les liens étroits avec l'histoire des migrations.

De George McManus à Bilal, en passant par Hugo Pratt, Uderzo et Goscinny, ou plus récemment Zeina Abirached ou Clément Baloup, nombre d'auteurs ont placé le thème de l'immigration au cœur de leur œuvre. C'est ce croisement méconnu entre deux histoires que le musée donne à voir à travers cette exposition itinérante.

\section{Un siècle d'histoires croisées}

Conçue pour une diffusion sous la forme de panneaux, cette exposition adopte une approche historique et artistique pour sensibiliser à ces histoires qui s'entremêlent. Adaptée aux néophytes comme aux amateurs de bande dessinée, elle est aussi un appel à la lecture de ces œuvres dont seuls quelques extraits sont présentés.

Une première série de panneaux propose une lecture générale de l'histoire de la bande dessinée au prisme des immigrations. Bien que née dans la presse européenne au XIXe siècle, la bande dessinée se développe aux États-Unis avec les funnies et les comics, ces historiettes humoristiques, souvent réalisées par des dessinateurs et graveurs d'origine européenne venus faire carrière aux États-Unis. À l'autre extrémité du continent américain, d'autres dessinateurs d'origine européenne inventent d'autres esthétiques, notamment avec l'École panaméricaine d'art fondée par Hugo Pratt et Alberto Breccia. En France, la bande dessinée se développe de Pilote à Fluide glacial grâce à des artistes, parfois éditeurs, dont l'histoire personnelle et le travail artistique sont marqués par l'immigration (Morris, Goscinny, Uderzo, Goetlib, puis Bilal, Baru, Boudjellal), ainsi que des artistes engagés pour une meilleure reconnaissance de la place des immigrés dans la société française (Reiser, Cabu...).

Une seconde série de panneaux revient sur les différents genres de bande dessinée, en croisant des œuvres récentes avec d'autres, plus anciennes. Des comics américains (Bringing up Father) à la science-fiction (Superman, Cité 14), le western (Texas Exil), ou encore le polar, l'immigration est 
née, hybrides, mêlant les genres traditionnels à d'autres pratiques artistiques (Là où vont nos pères, Petite histoire des colonies françaises).

Quatre reproductions de planches sont également présentées seules, pour une lecture simplement esthétique de la bande dessinée (Zeina Abirached, Vincent Zabus et Hippolyte, Baru, Julie Birmant et Clément Oubrerie).

À travers cette exposition, la bande dessinée apparaît à la fois comme un témoignage sensible de l'histoire de l'immigration, mais également comme un art dont le langage esthétique, conceptuel, est en perpétuelle transformation. L'immigration participe largement à ce processus, aux évolutions des langages artistiques, des récits et modes d'écriture. Alors qu'une révolution des outils de création s'opère progressivement, la BD demeure plus que jamais un art en mouvement, nourri des échanges et de la mobilité des hommes.

une thématique récurrente. Cette récurrence est amplifiée dans les œuvres croisant histoire collective et personnelle (autobiographie, biographie, autofiction), notamment depuis les travaux pionniers de Will Eisner autour du "roman graphique" dans les années 1970. Les auteurs de bande dessinée documentaire, genre plus récent popularisé par Joe Sacco, tâchent de faire comprendre les problématiques de notre monde contemporain et accordent de fait une grande place aux migrations. Pour certains auteurs, la bande dessinée est aussi un levier pour réveiller les consciences, alerter les lecteurs sur les injustices et les absurdités du monde contemporain, notamment les persécutions qui poussent à partir (Kurden People, Viva la Vida) ou qui touchent les migrants (Cauchemar blanc, Majic Majid).

Cette palette évoquant la diversité des formes esthétiques et artistiques de la bande dessinée se conclut avec les nouvelles formes de bande dessi-

\section{Une exposition gratuite, pensée pour tous et pour une large diffusion}

Le Musée national de l'histoire de l'immigration témoigne, à travers cette exposition itinérante, d'une politique partenariale hors les murs ambitieuse. D'abord, en adoptant un mode d'itinérance gratuit, rendu possible grâce au soutien de plusieurs partenaires financiers. Ensuite, en s'appuyant sur un réseau de partenaires diffuseurs permettant de démultiplier les lieux de présentation, de programmation complémentaire et les publics. Enfin, en faisant le pari d'une offre culturelle pour tous, destinée à faire changer les regards sur l'immigration et à lutter contre les préjugés et le racisme. L'enthousiasme et le soutien des partenaires financiers (la Fondation WFS, 


\section{INITIATIVES}

le département de la Seine-Saint-Denis, le conseil régional de la Guadeloupe), des maisons d'édition, des auteurs et des partenaires témoignent des attentes pour ce type d'offre culturelle.

En tout, vingt exemplaires de l'exposition seront diffusés en France et à l'international par l'Institut français, la Citéinternationale de la bande dessinée et de l'image, le département de la Seine-SaintDenis, l'Association des professeurs documentalistes de Guadeloupe, l'association Interbibly, la communauté d'agglomération Valence-Romans Sud Rhône-Alpes, la ville de Vitrolles, le Réseau Canopé, l'Association de la Ligue de l'enseignement Midi-Pyrénées, l'association Passage à l'Art, l'association Claje. Des partenariats sont à l'étude avec le conseil régional du Limousin et des partenaires d'île-de-France et du Nord-Pas-de-Calais.

Chacun de ces partenaires assurera l'itinérance gratuite d'un exemplaire de l'exposition auprès de son réseau, jusqu'en septembre 2020. Deux formats sont proposés, afin que l'exposition puisse être présentée dans des configurations différentes. Des territoires de la politique de la Ville aux institutions culturelles, en France métropolitaine, dans les territoires ultramarins ou à l'étranger, l'expositions'adresseraà des publics très différents. Sa conception s'est faite en conséquence, avec l'appui de professionnels de la médiation culturelle, de l'enseignement secondaire, ainsi que de la Cité internationale de la bande dessinée. De même, un soin particulier a été apporté aux ressources de communication et de médiation permettant aux structures d'accueil de se saisir de cette proposition le plus facilement possible (liens avec le programme scolaire et fiches pédagogiques, support de médiation, entretiens, bibliographie, liste d'intervenants possibles pour des ateliers d'auteurs).

Enfin, certaines présentations se feront sous des formes enrichies. Le Centre du patrimoine arménien, à Valence, présentera entre le 9 octobre 2015 et le 28 février 2016 une version "musée" de l'exposition, comprenant une scénographie ainsi que des œuvres originales prêtées par des artistes.

\section{/// Contact : itinerancebd@histoire-immigration.fr}

/// Voir également le catalogue de l'exposition : Albums, des histoires dessinées entre ici et ailleurs. Bande desssinée et immigration (1913-2013),Paris, Musée national de l'histoire de l'immigration/Futuropolis, octobre 2013, 26 euros. 